\title{
Clinical utility of antiemetics and complementary therapies in the prevention of postoperative nausea and vomiting
}

This article was published in the following Dove Press journal:

Clinical Audit

20 August 2013

Number of times this article has been viewed

\author{
Angela S Collins \\ Capstone College of Nursing, \\ University of Alabama, Tuscaloosa, AL, \\ USA; Baptist Princeton, Birmingham, \\ AL, USA
}

\begin{abstract}
Postoperative nausea and vomiting (PONV) is a persistent problem in perianesthesia practice. Approximately $30 \%$ of patients receiving anesthesia will experience this complication, which can increase patient dissatisfaction, increase costs, and in some patients precipitate other postoperative complications. During the last three decades, there has been increasing interest and research on best practices to diminish the incidence of this clinical problem, as patient satisfaction is linked to PONV guideline use. Some institutions have low incident rates because of consistent protocol use, and new medication classifications and multiple complementary therapies have been incorporated into daily use. There are several clinically useful guidelines and algorithms used to guide primary prevention strategies, as discussed in this review. However, severe PONV continues to be refractory to available interventions, and the best hope for elimination of this complication may arise from pharmacogenomics.
\end{abstract}

Keywords: postoperative nausea and vomiting, postanesthesia complications, clinical practice guidelines, pharmacology

\section{Introduction}

Nausea and vomiting are unpleasant and undesirable responses to illness and disease. Nausea and vomiting occurring with surgery produce two very different outcomes: the first is a situation that is self-limiting and uncomfortable, and that increases length of acute care stay; and the second is a potentially life-threatening set of consequences that can become very costly. ${ }^{1-3}$ Clinically problematic sequelae of postoperative nausea and vomiting (PONV) are: airway compromise; wound dehiscence; electrolyte imbalance; suture tension; esophageal tears; venous hypertension; surgical site bleeding; and increased intracranial pressure. ${ }^{2-4}$

PONV clinical manifestations are experienced by millions of patients annually worldwide, and exponentially increase the healthcare costs associated with surgery. ${ }^{1,5,6}$ Studies consistently report that if not treated prophylactically, $30 \%$ of surgical patients experience both postoperative nausea and vomiting. ${ }^{2,6-8}$ Patients receiving general anesthesia compared with regional anesthesia have an 11 -fold increase in PONV. ${ }^{9}$ Patients rank both nausea and vomiting as two of the top five most undesirable aspects of anesthesia and surgical care, and postoperative vomiting is ranked as the most unwanted complication of surgery. ${ }^{6,7,10-12}$ In one study, a patient survey revealed that patients were willing to pay increased out-of-pocket expenses to receive medications that would stop PONV. ${ }^{10}$ The psychological effects and distress associated with PONV can produce increased anxiety over future surgical procedures. ${ }^{12,13}$ 
The incidence of PONV has not decreased during the last 2 decades. This is confounding. In this time, two new categories of antiemetic medications specific to recently identified neurochemical trigger sites have been discovered, ${ }^{6}$ surgery has evolved to produce more minimally invasive surgical case types and decreased surgical procedure times, and anesthesia providers have changed the types of medications used in many surgeries in order to use both fewer anesthetic gases and more sedative medications with shorter half-lives. ${ }^{5}$ Researchers attribute the persistence in PONV incidence to a number of variables. One variable is that surgery has transformed to be primarily ambulatory; extensive and open surgical case types also encourage early mobilization and quicker discharge. ${ }^{5,14}$ Another variable is early home discharge and transport by car, as ambulatory surgery centers are sometimes located far from the patient's home. This transport may create motion sickness, another variable known to stimulate nausea and vomiting. Other recognized experts in this field of study state that the persistent rate of $30 \%$ of PONV is related to the wide genetic variability in metabolism of both anesthesia and antiemetic medications. ${ }^{14,15}$

The operational definition of PONV can vary widely between studies. For the purpose of this review, the PONV definition used is the one created by the American Society of PeriAnesthesia Nurses (ASPAN), which limits PONV to nausea and vomiting that occur within 24 hours of the surgical procedure. ${ }^{2}$

\section{Physiological basis of nausea and vomiting response}

To understand the mechanism of action of many pharmacological and alternative medicine interventions, a review of the neural and biochemical pathways associated with nausea and vomiting is required. Nausea and vomiting are two distinctly different physiological phenomena. Nausea is a subjective feeling and must be self-reported. It is experienced and interpreted by the patient and is present when the person reports the sensation. ${ }^{3,7}$ Nausea is closely linked to the olfactory sense (cranial nerve I). ${ }^{16-18}$ Other triggers listed in the literature are anxiety, vestibular stimulation, ocular surgery, and hormonal changes with birth control medications or pregnancy. ${ }^{13-15}$ Nausea is also associated with gastroparesis, adverse effects of opioid medications, and pain. Neuroscientists believe that nausea is a conscious cortical activity. However, the possible neural pathways that elicit nausea have not been identified (see Figure 1). ${ }^{16}$

There are, however, several well-outlined neural pathways that can stimulate a vomiting response. The central nervous system has two anatomical sites that initiate and regulate vomiting. The first is the chemoreceptor trigger zone (CTZ). The CTZ is located in the posterior aspect of the fourth ventricle floor with sensors in cerebrospinal fluid. Chemoreceptor cells are responsive to substances in both the blood and cerebrospinal fluid that are identified as toxins by this receptor site. ${ }^{13,14}$ The CTZ is not located within the blood-brain barrier. Five examples of CTZ triggers are viral infections, ingestion of chemicals, overeating, fear, and gastroparesis. The identified chemicals that have receptor sites in the CTZ are dopamine type 2, histamine type 1 , serotonin type 3 , neurokinin type 1 , and muscarinic cholinergic type $1 .{ }^{16}$ The $\mathrm{CTZ}$ also can be activated by the vestibular nerve (cranial nerve VIII) with dizziness, position change, motion sickness, and changes in air pressure. ${ }^{19}$

The second site that initiates and regulates vomiting is the medulla oblongata, which has afferent nerve inputs from the limbic system, pharyngeal, vagal, and midbrain. The limbic center is attributed to being the site at which anticipatory vomiting originates. Emotions generated within this region of the limbic system, such as anxiety, fear, and dread, can activate the medulla. ${ }^{13,14,16}$ Pharyngeal irritation resulting from instrumentation during surgery with endotracheal tubes, laryngeal mask airways, suction devices, or nasogastric tubes can stimulate the medulla. Vagal activation can occur with either intubation or suctioning of the upper and lower airway. Gastric distention and contraction can also influx vagal input to the medulla. Increased intracranial pressure is a well-known trigger of the medulla and is believed to originate from midbrain stimulation..$^{5,6}$

Vomiting has three phases in each cycle. Pre-ejection is started by the vagus nerve. This vagal stimulation increases salivation and causes bradycardia, the patient is diaphoretic, and there are alterations in the stomach tone. Some patients also experience dizziness and blurred vision resulting from diminished cardiac output because of parasympathetic activation. Ejection is initiated by diaphragmatic contraction and the opening of the gastric esophageal sphincter, followed by reflux of the gastrointestinal contents into the esophagus and reverse peristalsis, which ejects the contents from the mouth. The post-ejection phase is characterized by a subjective sensation of relief. This phase is also sometimes associated with a diminished sensation of nausea. ${ }^{3,5,19}$

\section{Patient-specific identified risk factors}

Nausea and vomiting rates differ among populations and surgical case types. This variability is attributed to the concept 
of threshold and risk factors. ${ }^{6,7}$ When two similar patients are exposed to similar noxious anesthesia stimuli, each patient will not exhibit the same PONV rate. This individual variation is described as a genetic set point or threshold for PONV. Risk factors can either be patient-specific or operative- and anesthesia-specific. $^{12}$

\section{Patient-specific risk factors}

Apfel et al $^{12}$ published a systematic review of the literature specific to the statistical likelihood that a risk factor will independently predict PONV. The strongest patient risk factor predictors were female gender, followed by history of PONV or motion sickness, being a nonsmoker, and a younger age. Females need a smaller stimulus for vomiting to occur and vomit two to three times more frequently than males of the same age. ${ }^{12}$ The origin of this risk factor is believed to be an endocrine mechanism. However, a robust randomized control trial of more than 5,000 patients found no significant statistical difference in PONV in women who were menstruating and those who were menopausal. ${ }^{20}$ Apfel et al write that the aspect of being female that contributes to PONV is unknown. ${ }^{12,20}$ Patient self-reporting of motion sickness or prior PONV is an indicator that there is a lower threshold for vomiting, as supported by a genome-wide associate study. In this study, the researchers found that intractable PONV ran in families. ${ }^{21}$ In addition, this study confirmed an association between a single nucleotide polymorphism and PONV susceptibility.

Anticipatory nausea and vomiting have a strong documented incidence as a learned response in patients receiving chemotherapy. It is unknown whether this response also occurs with PONV. If the patient has a belief or a genetically lower threshold for vomiting, this in many ways becomes a self-fulfilling prophecy. ${ }^{22}$ Two studies noted that the expectation of vomiting is identified as predictive of vomiting. However, the studied population comprised patients receiving chemotherapy, and it is not known whether the results can also be generalized to patients with PONV. High anxiety has a strong association with anticipatory vomiting as well. ${ }^{22}$ In addition, nonsmokers are at higher risk for postoperative nausea and vomiting; this factor has been validated by many risk factor studies. ${ }^{2-5,8,12}$ One hypothesis for why this occurs is that nicotine blocks liver enzymes that would increase the duration of anesthesia. Apfel et al attribute this characteristic of smokers as that "the protective effect of smoking may be due to functional changes in neuroreceptors from chronic exposure to nicotine, and thus nicotine withdrawal rather than nicotine exposure reduces smokers' susceptibility to PONV."12
There are two different observations about age. In children aged 3-16 years, there is an increasing incidence of PONV. There are only rare instances of PONV noted in children younger than 3 years. In adults, the incidence of PONV decreases with age in decades. Speculation about this variation revolves around age-related changes within the autonomic nervous system. ${ }^{12}$ Clinicians administering anesthesia write that the threshold for vomiting may require matured autonomic reflexes, and the effectiveness of these reflexes may diminish with age. ${ }^{6,16}$

\section{Anesthesia and operative risk factors}

Apfel et al's systematic review also noted that there are three powerful predictive risk factors that are associated with the operative and anesthesia process. ${ }^{12}$ The strongest anesthesia-related factors are use of volatile anesthetics, intraoperative and postoperative opioid use, and prolonged anesthesia time. ${ }^{12,14,20}$ Volatile anesthetics are often used to provide general anesthesia, and the mechanism of action of these gases is to decrease the level of arousal by diminishing the number and amplitude of action potentials in the central nervous system. Volatile gases enter the respiratory tract and quickly distribute throughout the body via the pulmonary capillary bed. ${ }^{19}$ The gases are sensed in the CTZ and may trigger the vomiting center. Nitric oxide is especially noted in research studies as being associated with a high risk for PONV when compared with other anesthetic gases. ${ }^{23}$ Theories for the emetogenic nature of nitrous are that the mechanism of action of this medication may act on dopamine receptors in the brain. In addition, nitrous may also change auditory pressures, leading to a vestibular disequilibrium known to contribute to vomiting. ${ }^{23}$

Opioids are usually concurrently administered during general anesthesia ${ }^{5}$ and are part of the general anesthesia prescription, an adjunct to prevent sympathetic stimulation resulting from pain. Opioids are also the primary drug classification of choice to treat postoperative pain. They work by binding to the mu and kappa receptors of the nervous system. These receptors are located in the brain within the rostral anterior cingulate cortex, as well as the brainstem. This receptor site activation diminishes the sensation of pain in the central nervous system. In the peripheral nervous system within the gut, mu receptor sites respond to opioid activation by decreasing peristalsis and promoting gastric distention. The gut receives additional irritation from the opioids that causes the enterochromaffin cells to release serotonin, which is another trigger, in the CTZ. ${ }^{2,5,24}$ 
Anesthesia time is a risk factor because PONV is doserelated; the longer the exposure to the anesthesia agents, the more likely a dose will accumulate that will cause vomiting activation. Sinclair et $\mathrm{a}^{24}$ describe a prevalence increase from $2.8 \%$ in patients whose surgery was less than 30 minutes, up to $27.7 \%$ in patients with a case time higher than 150 minutes. One recommendation from the Enhanced Recovery After Surgery (ERAS) Society is to use total intravenous anesthesia with intravenous dexamethasone and ondansetron. Two randomized control trials demonstrated a statistically significant reduction in PONV when total intravenous anesthesia was used. ${ }^{6}$

There are several studies that examine the importance of operative case type in predicting PONV. However, these studies are not as robust because of the smaller number of patients studied. One case type is neurosurgery patients: A systematic review by Neufeld and Newburn-Cook concludes that the reported incidence of nausea and vomiting in 13 studies of neurosurgery patients supported the use of prophylaxis in this operative case type. ${ }^{4}$ Postoperative vomiting is also strongly associated with endonasal transsphenoidal surgery for pituitary tumors. This surgical approach affects cerebral spinal fluid dynamics, and spinal fluid leaks and intraoperative lumbar drain use are also factors increasing the incidence of PONV. PONV is especially problematic in this case type because an increase in intracranial pressure (ICP) can trigger additional cerebral ischemia. ${ }^{3,5}$

A retrospective study of orthognathic surgery (corrective surgery to the jaw) including 514 patients found a prevalence of $40 \%$ in these surgical case types. ${ }^{19}$ The authors of this study attribute this higher incidence to the particular postoperative complications unique to this surgery. Examples of these clinical manifestations are lip and tongue numbness, orofacial swelling, and swallowing of bloody drainage. ${ }^{19}$ The jaw is often wired until the bones completely heal, which also mandates a change in diet texture and type that may contribute to increased nausea and vomiting. ${ }^{19}$

\section{Risk factor assessment tool}

Risk factor instruments are often used in the preoperative area to assess the probability that PONV will occur., ${ }^{8,1214}$ Apfel et al compared the validity of six models for PONV risk assessment. ${ }^{12}$ The clinical usefulness of the tool was related to the ease of use and correlation between the expected and actual rates of PONV. ${ }^{20}$ Kapoor et al compared two protocols for risk assessment of PONV and found no difference in the outcomes of the two groups. ${ }^{25}$ The three tools that are often cited in the literature are the ASPAN algorithm tools, Apfel simplified risk assessment tool, and Koivuranta's risk score. ${ }^{2,12,20}$ Each tool first identifies risk factors, weighs the strength of the risk factor, and suggests the number of multimodal interventions that must be completed to suppress PONV. These instruments are copyrighted and have been validated as clinically useful tools. Each tool recommends no interventions for PONV prevention for patients who are found to be low risk. Moderate risk should guide the clinician to choose a single intervention to prevent PONV. The highrisk group requires a multimodal interventional approach. ${ }^{14}$ Surgical service lines in many institutions use a multimodal approach to the prevention and treatment of PONV based on a combination of preference and risk stratification. ${ }^{3}$

\section{Clinical practice guidelines}

There are clinical practice guidelines from many respected entities that also include assessment tools. Three that are often cited are from the Society for AMBulatory Anesthesia (SAMBA), ASPAN, and the ERAS Society. 2,8,26,27 Table 1 contains additional details specific to the ASPAN guidelines. The clinical guidelines have six common threads that are clinically relevant. The first common thought is that universal treatment for all patients receiving anesthesia is a costly and unnecessary intervention. The second is that PONV prophylaxis is indicated for patients who are high risk and that this risk should be identified before surgery. Third is that some identified PONV risk factors have more of a predictive value than others. Fourth is that the more risk factors a patient is found to have by history, the more aggressive the multimodal approach should be. Fifth, if a patient has refractory PONV a different agent should be identified

Table I Recommendations from the American Association of PeriAnesthesia Nurses guidelines for postoperative nausea and vomiting prophylaxis risk assessment tool

\begin{tabular}{|c|c|c|}
\hline & Stratify level of risk & $\begin{array}{l}\text { Types of } \\
\text { prophylaxis } \\
\text { interventions }\end{array}$ \\
\hline $\begin{array}{l}\text { Document and } \\
\text { communicate patient } \\
\text { risk factors to the } \\
\text { anesthesiology and } \\
\text { operative team }\end{array}$ & $\begin{array}{l}\text { Low risk: no intervention } \\
\text { Moderate risk: one } \\
\text { prophylaxis intervention } \\
\text { Severe risk: two } \\
\text { prophylaxis interventions } \\
\text { Very severe risk: three } \\
\text { or more prophylaxis } \\
\text { interventions }\end{array}$ & $\begin{array}{l}\text { Anesthesia: } \\
\text { considerations use } \\
\text { of total intravenous } \\
\text { anesthesia and } \\
\text { regional blocks } \\
\text { Pharmacologic } \\
\text { considerations, } \\
\text { acupressure, } \\
\text { aromatherapy, } \\
\text { increased hydration }\end{array}$ \\
\hline
\end{tabular}

Notes: Information found within algorithm I: preoperative management of patients. ${ }^{8,26,27}$

Used with permission of ASPAN. American Society of PeriAnesthesia Nurses PONV/PDNV Strategic Work Team. ASPAN'S evidence-based clinical practice guideline for the prevention and/or management of PONV/PDNV.J Perianesth Nurs. 2006;2I (4):230-250. ${ }^{2}$ 
for pharmacological rescue. The final thread within each guideline acknowledges that alternate treatments such as acupuncture, aromatherapy, and herbal therapies have also been demonstrated to be effective in some patient populations.

Most PONV research studies are specifically designed to test the effects of a single type of treatment compared with another therapy. ${ }^{71115}$ The cost or effectiveness of different multimodal protocols has not been quantified by large-scale studies. There are several studies that isolate a particular surgical case type and that have used a multimodal approach to affect the overall incidence. There is no simple linear formula for interventions that lead to overall PONV suppression.

\section{Antiemetic options}

There are different levels of evidence that support the use of the following four primary types of interventions: pharmacologic methods; aromatherapy; herbal products; and acupressure and acupuncture in PONV. Timing of these interventions and matching the intervention to patient preference are also vital. ${ }^{3}$

\section{Pharmacologic methods}

No single medication classification has superior efficacy over another. Kranke et $\mathrm{al}^{14}$ use the following guideline for decision-making with the efficacy of these medications: when giving a single medication agent, the relative risk reduction is approximately $30 \%$.

There are nine pharmacological classifications of medications that assist the clinician with management of PONV: phenothiazines; antihistamines; prokinetic agents; serotonin type 3 receptor antagonists; neurokinin type 1 receptor antagonists; dronabinol, anticholinergic medications; corticosteroids; and selected mu two-site blockers.

The mechanism of action of phenothiazines is to block the dopamine 2 receptor sites in the CTZ. Decreased dopamine in the central nervous system promotes sedation, diminishes the sensation of nausea, and decreases the rate of vomiting. This medication classification is primarily used for its antipsychotic activity in dopamine-altered mental illness. ${ }^{28,29}$ It can lead to adverse effects, especially in the elderly, causing memory impairment and hallucinations. Phenothiazines are not typically first-line drugs for PONV but are used in the emergency room to treat migraine headaches and virusinduced nausea and vomiting.

Antihistamines work to block the histamine sites within the CTZ and also produce anticholinergic actions of dry mouth, tachycardia, and delayed gastric emptying. ${ }^{28,29}$ This medication classification is promoted as having the greatest efficacy in patients who have motion sickness. Timing of administration is most optimal when administered preoperatively $1-2$ hours before the triggering episode. . $^{3,28,29}$

Prokinetic agents work through antagonizing dopamine and increasing the release of acetylcholine. This medication classification works best with patients with diabetes and to blunt the adverse effects of mu activation of opioids. ${ }^{27,28}$ There is no dosing recommendation for the optimal timing of this medication.

Serotonin type 3 receptor antagonists are antagonists to serotonin receptors in the CTZ and work best when administered before induction or before the reversal of anesthetic agents.

Neurokinin type 1 receptor antagonists block these receptor sites in the medulla. This medication classification is used only with refractory PONV and is a rescue medication for chemotherapy-induced vomiting. ${ }^{28,29}$

Marinol ${ }^{\circledR}$ is the chemically active portion of the marijuana plant and is used as a medication for nausea and vomiting. The mechanism of action is not known. Marinol ${ }^{\circledR}$ has not been studied for its efficacy in PONV. ${ }^{28,29}$

Anticholinergic medications target the vestibular component of PONV. This medication classification is directed toward the acetylcholine receptors that innervate the vestibular input to the CTZ. Scopolamine is the medication in this classification that is most heavily used for patients with a history of motion sickness, ${ }^{28,29}$ and can be dispensed in a transcutaneous patch. This delivery method is slow release, and the patch can be left in place in the postoperative period. If the patient is particularly motion-sensitive, this treatment may aid in the reduction of PONV associated with ambulation in the postoperative phase..$^{6,27-29}$ The slow release of the anticholinergic medication necessitates that the patch be placed before surgery. It can be removed if the patient has difficultly with a persistent dry mouth or voiding after surgery.

Corticosteroids blunt the cortical response of anticipatory vomiting and block prostaglandin activity both centrally and peripherally. However, the specific mechanisms of action of corticosteroids are unknown. ${ }^{6,27,29}$ Corticosteroids are indicated for patients with the highest risk for PONV. The ERAS clinical practice guidelines advocate the use of corticosteroids to prevent PONV. ${ }^{27,30}$ In addition, three large studies have demonstrated a statistically significant difference in PONV treated with steroids versus PONV treated with placebo. ${ }^{6}$

Finally, selected mu two-site blockers block the gastroparesis induced by opioid use during the intraoperative and postoperative courses. These medications are indicated particularly for colon and rectal surgery case types. 
Many institutions have pharmacists who monitor and recommend the dosing and timing of mu receptor medications. ${ }^{31}$

\section{Aromatherapy}

The olfactory sense (cranial nerve I) can stimulate the CTZ. If this sensory nerve can be stimulated by the inhalation of aromas, the sensation of PONV is blunted. Because isopropyl alcohol packets are readily available in the surgical setting, nurses working in the operative and postoperative settings often use this therapy. ${ }^{17,18,32}$ Essential oils of peppermint and lavender are also reported as being an adjunct to other treatments to reduce PONV. ${ }^{33,34}$ Aromatherapy may distract the patient from fixation on the sensation of nausea and vomiting, as changing focus assists the patient in not attending to the triggers of PONV. ${ }^{2}$ The Cochrane Collaboration summarizes that the currently available research studies do not produce evidence to support the use of aromatherapy in PONV. ${ }^{35}$ However, in clinical practice, aromatherapy is an inexpensive intervention and has anecdotal support among clinicians.

The sense of smell is variable in each person, and some smells can be associated with allergies and traumatic emotional experiences. If a practitioner uses aromatherapy, it is vital to collect data on a patient's ability to smell, past experiences, and tolerance of the agents used. ${ }^{17,18}$

There also are commercially available aromatherapy products that have not been used in large-scale studies. ${ }^{34}$

\section{Herbal products}

Ginger is an herbal product that is often recommended for the treatment of nausea and vomiting. Ginger is used in some carbonated beverages and is often recommended by case report to blunt the sensation of nausea. The theory on the mechanism of action of ginger is that it works by reducing activation of the serotonin sites in the central nervous system and gastrointestinal system. ${ }^{36}$ However, ginger is also noted to prolong bleeding times by affecting platelet function. Because of this action on platelet aggregations, some anesthesia providers believe that ginger is contraindicated in PONV treatment. Patients with diabetes have also been noted as having potentiation of antidiabetic medications with ginger, leading to hypo- and hyperglycemic responses. Morin et al reported that treatment with ginger was not an effective therapy in PONV. ${ }^{37}$ A current systematic review by Ding et al reports that ginger is a safe and effective treatment for pregnancy-induced nausea and vomiting. ${ }^{38}$ At the conclusion of this study, however, these nurse-midwives express concern about three persistent questions about the use of ginger: what is the maximal dosage that is safe for the mother-child dyad? What is the effect of potential drug-herb interactions? Are there rescue strategies and consequences of ginger overdosage?

\section{Acupressure and acupuncture}

Acupressure and acupuncture are the most widely researched complementary treatments for PONV. The area for acupressure is located in the wrist and is described as the P6 meridian. There are different methods to perform acupressure, both noninvasive and invasive. Noninvasive methods are manual pressure, use of a peripheral nerve stimulator with train of four, and placing a wristband with a compressive device over the P6 site. Invasive methods are the use of needles in the peripheral nerve. The Cochrane Collaboration systematic review looked at more than 40 studies that used acupressure as an adjunct to management of PONV. ${ }^{39}$ The executive summary stated that after examining the evidence, there was a statistically significant decrease in rates of PONV using acupressure. ${ }^{39}$ One study on cardiovascular surgical patients found not only that the incidence of PONV decreased in the treatment group but also that the effects extended to 48 hours into the postoperative phase..$^{40}$

\section{Effect on patient management and healthcare resources}

PONV affects healthcare costs in four ways: PONV can result in an unexpected hospitalization of an ambulatory surgery patient; can prolong the time needed for patient monitoring in the postanesthesia area and critical care unit; may increase the overall hospital stay for a surgical patient; and may delay the recovery of the patient who experienced a PONV-induced delay in returning to work and in resumption of normal activities of daily living. ${ }^{14,15}$

There is consensus that PONV prophylaxis is needed and that it must be cost-effective, particularly in patients who have multiple risk factors. Dzwonczyk et al report that "the cost of PONV prophylaxis is economically beneficial for the hospital when weighed against the expenses generated by treating patients returning to the hospital with PONV." ${ }^{15}$ Each episode of vomiting extends time needs in postanesthesia recovery by about 30 minutes. ${ }^{2,5}$

\section{Multimodal bundling of PONV interventions}

Changes in practice can be suggested by evidence from clinical practice or mandated by regulations and reimbursement. ${ }^{15}$ PONV rates are often internally reviewed in hospitals but are not published on websites or listed on 
consent documents. The question then becomes what the motivators and obstacles are for clinicians to implement specific interventions. Patient perceptions with healthcare quality are routinely measured in the Hospital Care Quality Information from the Consumer Perspective survey, ${ }^{41}$ which is linked to Medicare and Medicaid reimbursement. Three of the survey question areas could link back to PONV rates. The first area is the two questions in which the patient is asked, "during this hospital stay, how often did nurses listen carefully to you?"41 The same question is also asked with the word "doctors" used as the subject. The next area is three questions specific to pain, asking whether the patient's pain was controlled and whether the hospital staff did everything they could to help the patient with pain. The final perception question that could also link to PONV rates is the question on medication adverse effects, as PONV is considered an adverse effect of anesthesia. ${ }^{41}$ The patient perception surveys are bottom-line motivators for PONV in the hospital environment.

One of the most commonly discussed obstacles to improvement in healthcare outcomes is interdisciplinary communication, ${ }^{42}$ and communication is cited as the most frequent cause of problems in the clinical care process. ${ }^{42}$ There are multiple healthcare providers involved with assessment, monitoring, and prevention of PONV, each with their own viewpoint. At this time, in each area in which anesthesia care is provided, each interdisciplinary group choses their own protocols, champions, and processes to outline an approach to combat PONV. The level of engagement within each discipline and the monitoring of the outcome are variables cited as producing optimal outcomes. ${ }^{42}$ An example of an interdisciplinary protocol that has been recently published is Myklejord et al, in which the authors describe a $5 \%$ decrease in PONV in their surgical patient population. ${ }^{43}$ These results occurred after the authors' institution developed a framework of interventions that were triggered to manage preoperative antiemetic prophylaxis. The patients who had three or more risk factors for PONV remained refractory to outlined interventions. ${ }^{43}$

Bundling of interventions for other clinical problems is in widespread use for surgical improvement projects. Bundling is a method of clinical problem solving intended to exponentially improve outcomes, as interventions that are clustered interrupt an undesired outcome at multiple sites in

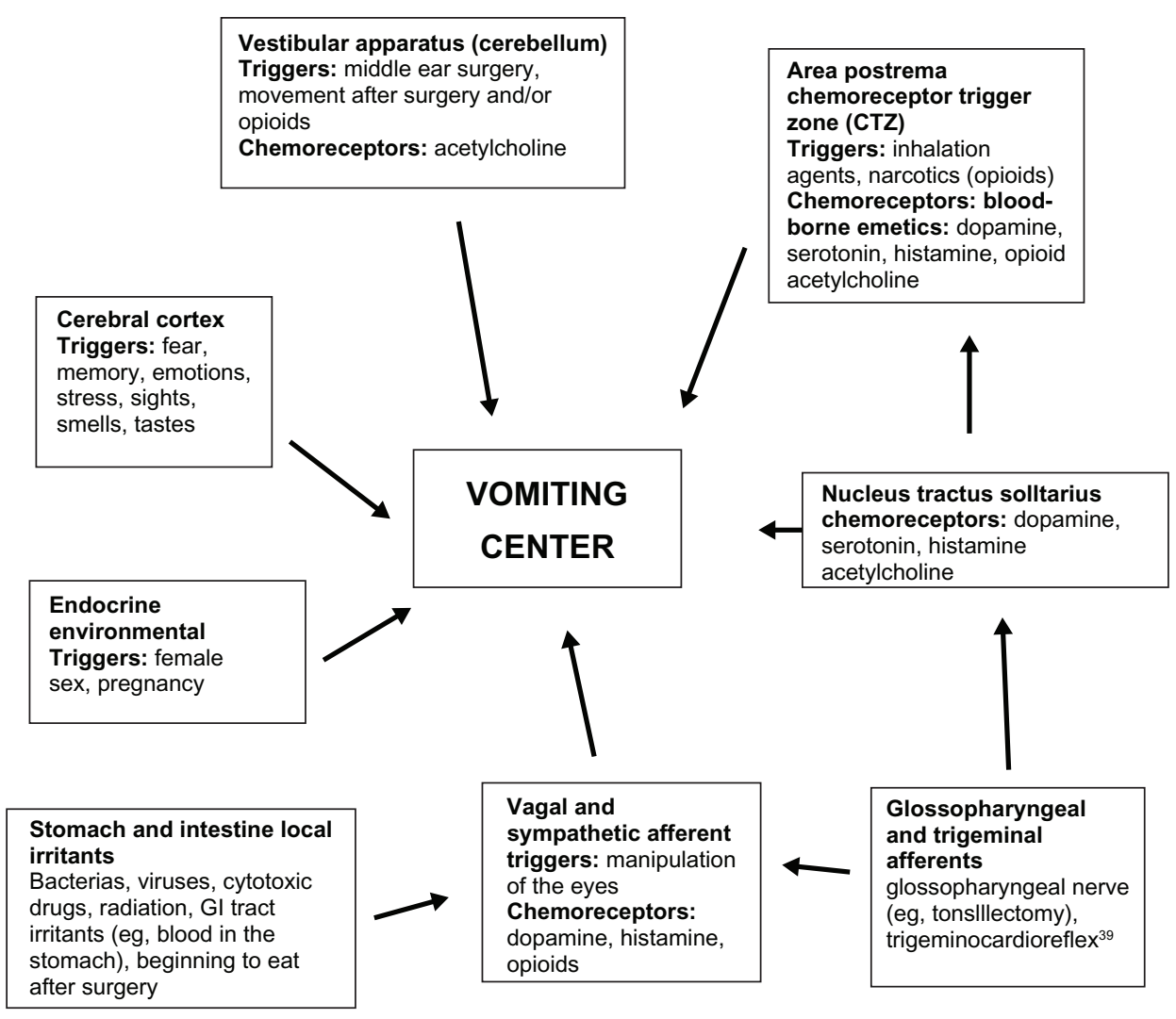

Figure I Mechanism and neurotransmitter system of PONV.

Note: Used with permission of Elsevier. Silva AC, O'Ryan F, Poor DB. Postoperative nausea and vomiting (PONV) after orthognathic surgery: a retrospective study and literature review. J Oral Maxillofac Surg. 2006;64(9):1 385-1397.19 
the healthcare process. An example of bundled interventions is the 100,000 Lives Campaign's six practices, one of which was to prevent surgical site infections. ${ }^{44}$ Embedded within the campaign's consensus guidelines are algorithms that recommend matching the assessed risk with increasing numbers of multimodal interventions. Implementation of these bundles of interventions is intended to save lives; however, the outcomes of preventing death are hard to quantify, and the actual improved outcomes and decreased mortality rate are hard to link as cause and effect. ${ }^{44}$

Each of the guidelines by ASPAN, SAMBA, and the ERAS Society, provide a framework of tools to identify highrisk patients and a series of bundled interventions that have evidence to support their use in the perioperative/perianesthesia setting. Apfel et al's current systematic review is an excellent example of determining the power of each risk factor for triggering PONV. ${ }^{12}$ The largest gap in the current research literature is in qualitative and quantitative data that delineate the most effective interventions to prevent PONV.

\section{Genetics}

Pharmacogenomics is a new discipline of study that links genetics and the process of drug absorption, distribution, metabolism, and excretion in the body. Genetics is heralded as the future of individualized interventions for disease. ${ }^{45}$ As Candiotti succinctly stated in the title of his article, anesthesia and genetics are not ready for prime time. ${ }^{46}$ At this time, the best methods for studying the links between genetics, drugs, and diseases are still unfolding. The rationale for using PONV as an object of genetic research is because PONV has been noted in patient histories to be familial.

Janicki's study gives evidence of the potential association of genetics within patients with severe nausea and vomiting. ${ }^{21}$ As receptor sites are important in drug metabolism, and these sites are genetically determined, several studies have been conducted during the last decade. This author identified genetic studies that have been undertaken by seven additional researchers specific to a particular medication's effect and the genetic variations of a receptor. ${ }^{46-52}$ However, until genetics can assist the clinician with optimal interventions to match with the patient's PONV clinical presentation, the research lacks clinical efficacy.

\section{Conclusion}

PONV continues to be a persistent problem after general anesthesia. Practicing clinicians should examine the different clinical practice guidelines available that have a goodness of fit for their patient populations. Consistent assessment of patients to identify those who have high risk is a prudent strategy. Designing a plan of care specific to each patient's assessment and then tailoring the interventions to achieve the best outcome requires a multimodal approach of all of the care providers in the perioperative setting. The highestrisk patients need a combination of interventions with different medication classifications to suppress PONV. Most guidelines recommend the practice of bundling interventions for PONV. Using two different medication classifications and adding complementary therapies is discussed in many clinical practice guidelines. The greatest area of promise in this area of research is the identification of the genetic variables that predispose patients to a greater risk for PONV. Pharmacogenomics gives the clinician hope that one day, identification of tailored anesthesia prescriptions and antiemetic protocols will be possible.

\section{Disclosure}

The authors report no conflicts of interest in this work.

\section{References}

1. Gan TJ. Risk factors for postoperative nausea and vomiting. Anesth Analg. 2006;102(6):1884-1898.

2. American Society of PeriAnesthesia Nurses PONV/PDNV Strategic Work Team. ASPAN'S evidence-based clinical practice guideline for the prevention and/or management of PONV/PDNV.J Perianesth Nurs. 2006;21(4):230-250.

3. Collins AS. Postoperative nausea and vomiting in adults: implications for critical care. Crit Care Nurse. 2011;31(6):36-45.

4. Neufeld SM, Newburn-Cook CV. What are the risk factors for nausea and vomiting after neurosurgery? A systematic review. Can J Neurosci Nurs. 2008;30(1):23-34.

5. Odom-Forren J. Drain's Perianesthesia Nursing: A Critical Care Approach, 6th ed. St Louis: Saunders, Elsevier; 2012.

6. Smith HS, Smith EJ, Smith BR. Postoperative nausea and vomiting. Ann Palliat Med. 2012;1(2):94-102.

7. Apfel CC, Roewer N, Korttila K. How to study postoperative nausea and vomiting. Acta Anaesthesiol Scand. 2002;46(8):921-928.

8. Apfel CC, Kranke P, Eberhart LH. Comparison of surgical site and patient's history with a simplified risk score for the prediction of postoperative nausea and vomiting. Anaesthesia. 2004;59(11): 1078-1082.

9. Apfel CC, Stoecklein K, Lipfert P. PONV: a problem of inhalational anaesthesia? Best Pract Res Clin Anaesthesiol. 2005;19(3):485-500.

10. Kerger H, Turan A, Kredel M, et al. Patients' willingness to pay for anti-emetic treatment. Acta Anaesthesiol Scand. 2007;51(1):38-43.

11. Dolin SJ, Cashman JN, Bland JM. Effectiveness of acute postoperative pain management: I. Evidence from published data. Br J Anaesth. 2002;89(3):409-423.

12. Apfel CC, Heidrich FM, Jukar-Rao S, et al. Evidence-based analysis of risk factors for postoperative nausea and vomiting. Br J Anaesth. 2012;109(5): 742-753.

13. McCaffrey R. Make PONV prevention a priority. OR Nurse. 2007;1(2): $39-45$.

14. Kranke P, Schuster F, Eberhart LH. Recent advances, trends and economic considerations in the risk assessment, prevention and treatment of postoperative nausea and vomiting. Expert Opin Pharmacother. 2007;8(18):3217-3235. 
15. Dzwonczyk R, Weaver TE, Puente EG, Bergese SD. Postoperative nausea and vomiting prophylaxis from an economic point of view. Am J Ther. 2012;19(1):11-15.

16. Story L. Pathophysiology. Sudbury, MA: Jones and Bartlett; 2012.

17. Doty RL. Sense of smell. In: Ramachandran VS, editor. Encyclopedia of Human Behavior, 2nd ed. London: Academic Press, Elsevier; 2012:366-372.

18. Stockhorst U, Pietrowsky R. Olfactory perception, communication, and the nose-to-brain pathway. Physiol Behav. 2004;83(1):3-11.

19. Silva AC, O'Ryan F, Poor DB. Postoperative nausea and vomiting (PONV) after orthognathic surgery: a retrospective study and literature review. J Oral Maxillofac Surg. 2006;64(9):1385-1397.

20. Apfel CC, Korttila K, Abdalla M, et al; IMPACT Investigators. A factorial trial of six interventions for the prevention of postoperative nausea and vomiting. N Engl J Med. 10, 2004;350(24):2441-2451.

21. Janicki PK, Vealey R, Liu J, Escajeda J, Postula M, Welker K. Genome-wide association study using pooled DNA to identify candidate markers mediating susceptibility to postoperative nausea and vomiting. Anesthesiology. 2011;115(1):54-64.

22. Middleton J, Lennan E. Effectively managing chemotherapy-induced nausea and vomiting. Br J Nurs. 2011;20(17):S7-S8, S10-S12, S14-S15.

23. Myles PS, Leslie K, Chan MT, et al; ENIGMA Trial Group. Avoidance of nitrous oxide for patients undergoing major surgery: a randomized controlled trial. Anesthesiology. 2007;107(2):221-231.

24. Sinclair DR, Chung F, Mezei G. Can postoperative nausea and vomiting be predicted? Anesthesiology. 1999;91(1):109-118.

25. Kapoor R, Hola ET, Adamson RT, Mathis AS. Comparison of two instruments for assessing risk of postoperative nausea and vomiting Am J Health Syst Pharm. 2008;65(5):448-453.

26. Gan TJ, Meyer TA, Apfel CC, et al; Society for Ambulatory Anesthesia. Society for Ambulatory Anesthesia guidelines for the management of postoperative nausea and vomiting. Anesth Analg. 2007;105(6): 1615-1628.

27. Varadhan KK, Neal KR, Dejong CH, Fearon KC, Ljungqvist O, Lobo DN. The enhanced recovery after surgery (ERAS) pathway for patients undergoing major elective open colorectal surgery: a meta-analysis of randomized controlled trials. Clin Nutr. 2010;29(4): 434-440.

28. Geralyn F, Smith S, Abrams AC. Clinical Drug Therapy: Rationales for Nursing Practice, 10th ed. Philadelphia: Wolter Kluwer Health, Lippincott; 2014.

29. Brunton LL, Chabner BA, Knollman BC. Goodman and Gilman's The Pharmacological Basis of Therapeutics, 12th ed. New York: McGrawHill; 2010.

30. http://www.erassociety.org/ [homepage on the Internet]. ERAS Society. Accessed July 18, 2013.

31. Bates JJ, Foss JF, Murphy DB. Are peripheral opioid antagonists the solution to opioid side effects? Anesth Analg. 2004;98(1):116-122.

32. Johnson JV, Grubb GS, Constantine GD. Endometrial histology following 1 year of a continuous daily regimen of levonorgestrel 90 micro g/ethinyl estradiol 20 micro g. Contraception. 2007;75(1):23-26.

33. Krueger DD, Tuffy LP, Papadopoulos T, Brose N. The role of neurexins and neuroligins in the formation, maturation, and function of vertebrate synapses. Curr Opin Neurobiol. 2012;22(3):412-422.

34. King L, Ragan S, Thomason HC, Clement F, Botchuck J, Hardin S. Quease Ease aromatherapy for the treatment of PONV [webpage on the Internet]. American Association of Critical Care Nurses. Available from: http://www.aacn.org/wd/ntispeakermats/PosterPresentations/00037430/ index.html. Accessed July 18, 2013.
35. Hines S, Steels E, Chang A, Gibbons K. Aromatherapy for treatment of postoperative nausea and vomiting. Cochrane Database Syst Rev. 2012;4:CD007598.

36. Chaiyakunapruk N, Kitikannakorn N, Nathisuwan S, Leeprakobboon K, Leelasettagool $\mathrm{C}$. The efficacy of ginger for the prevention of postoperative nausea and vomiting: a meta-analysis. Am J Obstet Gynecol. 2006;194(1):95-99.

37. Morin AM, Betz O, Kranke P, Geldner G, Wulf H, Eberhart LH. Ist Ingwer ein sinnvolles Antiemetikum für die postoperative Phase? [Is ginger a relevant antiemetic for postoperative nausea and vomiting?]. Anasthesiol Intensivmed Notfallmed Schmerzther. 2004;39(5):281-285. German.

38. Ding M, Leach M, Bradley $H$. The effectiveness and safety of ginger for pregnancy-induced nausea and vomiting: a systematic review. Women Birth. 2013;26(1):e26-e30.

39. Lee A, Fan LT. Stimulation of the wrist acupuncture point P6 for preventing postoperative nausea and vomiting. Cochrane Database Syst Rev. 2009;(2):CD003281.

40. Korinenko Y, Vincent A, Cutshall SM, Li Z, Sundt TM 3rd. Efficacy of acupuncture in prevention of postoperative nausea in cardiac surgery patients. Ann Thorac Surg. 2009;88(2):537-542.

41. http://www.hcahpsonline.org [homepage on the Internet]. Baltimore, MD: Centers for Medicare and Medicaid Services. Accessed June 13, 2013.

42. The Joint Commission. Sentinel Event Data Root Causes by Event Type 2004-2Q 2012. Available from http://www.jointcommission. org/assets/1/18/Root_Causes_Event_Type_2004_2Q2012.pdf. 2012. Accessed June 13, 2013.

43. Myklejord DJ, Yao L, Liang H, Glurich I. Consensus guideline adoption for managing postoperative nausea and vomiting. WMJ. 2012;111(5):207-213

44. Wachter RM, Pronovost PJ. The 100,000 Lives Campaign: A scientific and policy review. Jt Comm J Qual Patient Saf. 2006;32(11): 621-627.

45. Frandsen G, Smith SS. Abrams' Clinical Drug Therapy, 10th ed. Philadelphia: Wolters Kluwer| Lippincott Williams \& Wilkins.

46. Candiotti K. Anesthesia and pharmacogenomics: not ready for prime time. Anesth Analg. 2009;109(5):1377-1138.

47. Candiotti KA, Birnbach DJ, Lubarsky DA, et al. The impact of pharmacogenomics on postoperative nausea and vomiting: do CYP2D6 allele copy number and polymorphisms affect the success or failure of ondansetron prophylaxis? Anesthesiology. 2005;102(3):543-549.

48. Nielsen M, Olsen NV. Genetic polymorphisms in the cytochrome P450 system and efficacy of 5-hydroxytryptamine type 3 receptor antagonists for postoperative nausea and vomiting. Br J Anaesth. 2008;101(4):441-445.

49. Kaiser R, Sezer O, Papies A, et al. Patient-tailored antiemetic treatment with 5-hydroxytryptamine type 3 receptor antagonists according to cytochrome P-450 2D6 genotypes. J Clin Oncol. 2002;20(12): 2805-2811.

50. Kaiser R, Tremblay PB, Sezer O, Possinger K, Roots I, Brockmöller J. Investigation of the association between 5-HT3A receptor gene polymorphisms and efficiency of antiemetic treatment with 5-HT3 receptor antagonists. Pharmacogenetics. 2004;14(5):271-278.

51. Rueffert H, Thieme V, Wallenborn J, et al. Do variations in the 5-HT3A and 5-HT3B serotonin receptor genes (HTR3A and HTR3B) influence the occurrence of postoperative vomiting? Anesth Analg. 2009;109(5): 1442-1447.

52. Nakagawa M, Kuri M, Kambara N, et al. Dopamine D2 receptor Taq IA polymorphism is associated with postoperative nausea and vomiting. $J$ Anesth. 2008;22(4):397-403. 
Clinical Audit

Dovepress

\section{Publish your work in this journal}

Clinical Audit is an international, peer-reviewed, open access journal focusing on the processes and outcomes of clinical audit in any area of healthcare. All aspects of patient care are addressed within the journal and practitioners from all disciplines are invited to submit their work. Areas covered include: Publication of audits; How an audit has changed practice; changed patient care; Calls and justifications for new audits. The manuscript management system is completely online and includes a very quick and fair peer-review system, which is all easy to use. Visit http://www.dovepress. com/testimonials.php to read real quotes from published authors. 